V. Mandrekar

Nagoya Math. J.

Vol. 54 (1974), 69-78

\title{
ON THE MULTIPLE MARKOV PROPERTY OF LÉVY-HIDA FOR GAUSSIAN PROCESSES
}

\section{MANDREKAR*}

The purpose of this note is to clarify relations between multiple Markov properties (MMP) defined by Lévy ([8], [9]) and Hida [5] for Gaussian processes and to extend some work in Lévy [8] and Hida [5]. In the stationary Gaussian case it has been shown ([5], [4]) that these notions of MMP coincide. Interesting examples of (non-stationary) processes satisfying MMP can be found in [5], [8]. We now set up some notation: Let $\{x(t), t \in \boldsymbol{R}\}$ be a Gaussian stochastic process (GSP) and $\mathscr{F}_{t}$ for each $t$ be the $\sigma$-field generated by $\{x(\tau), \tau \leq t\}$. (Henceforth, $\mathscr{F}_{t}=$ $\sigma\left\{x_{\tau}, \tau \leq t\right\}$.) We denote by $Y(t, s)=E\left(x(t) \mid \mathscr{F}_{s}\right)$ the conditional expectation of $x(t)$ given $\mathscr{F}_{s}$. We note that $Y(t, s)$ is the orthogonal projection of $x(t)$ onto $H(x: s)$ where $H(x: s)=\subseteq\{x(\tau), \tau \leq s\}^{1)}$. A GSP is said to be $N$-ple Markov ([5], p. 128) if for each $s$ fixed $Y\left(t_{i}, s\right)$ are linearly independent (as elements of the vector space of square integrable functions) for $s \leq t_{1}<t_{2}<\ldots<t_{N}$ and $Y\left(t_{i}, s\right)$ are linearly dependent for $t_{i}(i=1,2, \cdots, N+1)$ satisfying $s<t_{1}<\cdots<t_{N+1}$. Our first result is to show that if $\mathscr{F}_{t-0}=\mathscr{F}_{t}$ (or equivalently $H(x: t)=H(x: t-0)$ ). Then there exists an $N$-dimensional Martingale Gaussian process $\{u(t), t \in \boldsymbol{R}\}$ and a non-random family of functions $\left\{f_{i}(t)\right\}_{i=1}^{N}$ such that

$$
x(t)=\sum_{i=1}^{N} f_{i}(t) u_{i}(t),
$$

where (i) $\operatorname{det}\left\{f_{i}\left(t_{j}\right)\right\} \neq 0$ for any $t_{1}<t_{2}<\cdots t_{N}$.

(ii) for all $t,\left\{u_{1}(t), \cdots, u_{N}(t)\right\}$ is linearly independent set.

(0.2) (iii) $\underline{u}(\cdot)=\left(u_{1}(\cdot), \cdots, u_{N}(\cdot)\right)^{*}$ is an $N$-variate Martingale, that is $E\left(u_{i}(t) \mid \mathscr{F}_{s}(\underline{u})\right)=u_{i}(s)$ with $\mathscr{F}_{s}(\underline{u})=\sigma\left\{u_{i}(\tau), \tau \leq s, i=1,2, \cdots, N\right\}$, $i=1,2, \cdots, N, s \leq t$.

Received November 9, 1973.

* Supported in part by NSF GP-28658.

1) $S\{\}$ denotes the subspace generated by \{\} . 
(iv) $\mathscr{F}_{t}(x)=\mathscr{F}_{t}(\underline{u})$ (or equivalently, $H(x: t)=H(\underline{u}: t)$ with $H(\underline{u}: t)$ $\left.=\Im\left\{u_{i}(\tau), \tau \leq t, i=1,2, \cdots, N\right\}\right)$.

This representation ${ }^{2)}$ will be referred to as Lévy representation in view of Lévy's definition ([9], [8], p. 158) of Markovian process of exactly order $N$. We show that such a representation is unique. This representation can be used to construct $N$-ple Markov process of multiplicity $N$, which answers a question by Lévy [9] regarding multiplicity of such processes. The representation (0.1) is called proper if $(0.2)$ (iv) is satisfied. We examine the question regarding properness of the representation (0.1). These results include some earlier work of Lévy ([8], [9]) and Hida [5]. Finally, we give an interesting necessary and sufficient condition for a differential operator to be factorable in terms of the multiple Markov property of the solution of an associated stochastic differential equation.

During the preparation of this work the author had the privilege of extensive discussions with Professor T. Hida whose suggestions led to considerable improvement in content and presentation of the original results announced in the preprint [11].

1. Let $\{\underline{u}(t), t \in \boldsymbol{R}\}$ be an $N$-variate GSP and for each $t$, let $\mathscr{F}_{t}(\underline{u})=$ $\sigma\left\{u_{i}(\tau), \tau \leq t, i=1,2, \cdots, N\right\}$. We say that $\{\underline{u}(t), t \in \boldsymbol{R}\}$ is an $N$-variate Martingale if $E\left(u_{i}(t) \mid \mathscr{F}_{s}(\underline{u})\right)=E\left(u_{i}(t) \mid \underline{u}(s)\right)=u_{i}(s), i=1,2, \cdots, N$. We now state our main representation theorem.

1.1 TheoRem. Let $\{x(t), t \in \boldsymbol{R}\}$ be an $N$-ple Markov GSP satisfying $\mathscr{F}_{t}=\mathscr{F}_{t-0}(x)=\sigma\left(\bigcup_{n} \mathscr{F}_{t-1 / n}(x)\right)$, then there exists an $N$-variate Martingale $G S P\{\underline{u}(t), t \in \boldsymbol{R}\}$ such that the GSP $x$ has representation (0.1) satisfying (0.2).

Proof. Let $s<\tau$, then for $s \leq t_{1}<t_{2}<\cdots<t_{N}<\tau$ we get

$$
Y(\tau, s)=\sum_{i=1}^{N} a_{i}\left(\tau, t_{1}, \cdots, t_{N}\right) Y\left(t_{i}, s\right) .
$$

Also for $s \leq s_{1}<s_{2}<\ldots<s_{N}<t_{1}<\ldots<t_{N}$ we get

$$
Y(\tau, s)=\sum_{j=1}^{N} \sum_{k=1}^{N} a_{k}\left(\tau, t_{1}, \cdots, t_{N}\right) a_{j}\left(t_{k}, s_{1}, \cdots, s_{N}\right) Y\left(s_{j}, s\right) .
$$

From (1.2), (1.3) and linear independence of $\left\{Y\left(s_{j}, s\right)\right\}_{j=1}^{N}$ and $\left\{Y\left(t_{k}, s\right)\right\}_{k=1}^{N}$ we have

2) Originally this representation was obtained by using the multiplicity theory [5]. The present concise and improved proof was suggested by Professor Hida and Nomoto. 


$$
\left\{\begin{array}{l}
\text { (i) } \sum_{k=1}^{N} a_{k}\left(\tau, t_{1}, \cdots, t_{N}\right) a_{j}\left(t_{k}, s_{1}, \cdots, s_{N}\right)=a_{j}\left(\tau, s_{1}, \cdots, s_{N}\right) \\
\text { (ii) } \operatorname{det}\left(\left(a_{j}\left(t_{k}, s_{1}, \cdots, s_{N}\right)\right)\right) \neq 0 .
\end{array}\right.
$$

Following notation and argument as in Hida ([5], pp. 129-130) we get that $f_{s}(\tau)=a(\tau, s) B(s, t)$ has an extension independent of $s$. We denote it by $\underline{f}(\tau)=\left(f_{1}(\tau), \cdots, f_{n}(\tau)\right)$. In view of (1.4) (ii) $\left\{f_{i}(\tau)\right\}_{i=1}^{N}$ satisfies condition (0.1) (i)

(1.5) $Y(\tau, s)=a(\tau, t) Y(t, s)=\underline{f}(\tau) B^{-1}(s, t) Y(t, s)=\underline{f}(\tau) u^{* 3)}(s, s, t), s<\tau$.

For $\tau>t_{N}^{\prime}>\ldots>t_{1}^{\prime}>s_{N}^{\prime}>\ldots>s_{1}^{\prime}$

$$
Y(\tau, s)=\underline{f}(\tau) u^{*}\left(s, \underline{s}^{\prime}, \underline{t}^{\prime}\right) .
$$

Now (1.5), (1.6) and condition (1.4) (ii) imply $u\left(s, \underline{s}^{\prime}, \underline{t}^{\prime}\right)=u(s, s, t)$, $\tau>\max \left(t_{N}^{\prime}, t_{N}\right)$, i.e., $\underline{u}(s)=\underline{u}(s, \underline{s}, t)$ is well defined as a function $s$. We thus get for $s<\tau$

$$
E\left(x(\tau) \mid \mathscr{F}_{s}\right)=\sum_{i=1}^{N} f_{i}(\tau) u_{i}(s)
$$

Clearly, for each $i,\left\{u_{i}(s), \mathscr{F}_{s}(x), s<\tau\right\}$ is a Martingale giving for each $i, E\left|u_{i}(s)\right|^{2}$ is non-decreasing. Also using (1.7), (0.2) (i) and Jenssen inequality for conditional expectation we get for $s<\tau, \sum_{i=1}^{N} \sup _{s<\tau} E\left|u_{i}(s)\right|^{2}$ is finite. By Martingale convergence theorem ([3], Ch. VII) for each $i$, a.e. limit of $u_{i}(s)$ as $s \uparrow \tau$ exists and if we denote by $u_{i}(\tau)=\lim _{s \uparrow \tau} u_{i}(s)$ we get $\left\{u_{i}(s), \tilde{\mathscr{F}}_{s}, s \leq \tau\right\}$ is a Martingale where $\tilde{\mathscr{F}}_{\tau}=\sigma\left(\bigcup_{s<\tau} \mathscr{F}_{s}\right)$ and $\tilde{\mathscr{F}}_{s}$ $=\mathscr{F}_{s}$ for $s<\tau$. But by assumption $\tilde{\mathscr{F}}_{\tau}=\mathscr{F}_{\tau}$ and hence $\left\{u_{i}(s), \mathscr{F}_{s}, s \leq \tau\right\}$ is a Martingale for each $i$. Taking in (1.7) lim as $s \uparrow \tau$ on both sides we get for all $\tau$

$$
x(\tau)=E\left(x(\tau) \mid \mathscr{F}_{\tau-0}\right)=\sum_{i=1}^{N} f_{i}(\tau) u_{i}(\tau) .
$$

By the construction, we get $\mathscr{F}_{t}(\underline{u}) \subseteq \mathscr{F}_{t}(x)$ for each $t$. By $(1.8), \mathscr{F}_{t}(x)$ $\subseteq \mathscr{F}_{t}(\underline{u})$ giving (0.2) (iv). Linear independence of $\left\{u_{i}(s), i=1,2, \cdots, N\right\}$ for each $s$ follows from (1.7), $N$-ple Markov property and the proved property $(0.2)$ (i) of the $f_{i}^{\prime}$ 's.

1.9 Remark. We note that although property $\mathscr{F}_{t}(x)=\mathscr{F}_{t-0}(x)$ is required for the Lévy representation of $N$-ple Markov processes, the

\footnotetext{
3) Denotes the transpose.
} 
GSP of the form (0.1) satisfying (0.2) is always $N$-ple Markov.

With this in mind we state the following

1.10 THEOREM. A GSP $\{x(t), t \in R\}$ of the form (0.1) satisfying (0.2) is N-ple Markov. In this case, the functions $f_{i}$ 's and the process $\{\underline{u}(t), t \in \boldsymbol{R}\}$ are unique in the sense that if $x$ has representation (0.1) with functions $f_{i}^{\prime}(i=1,2, \cdots, N)$ and a process $\left\{\underline{u}^{\prime}(t), t \in \boldsymbol{R}\right\}$ satisfying (0.2) then there exists a non-singular constant matrix $K=\left(\left(k_{i j}\right)\right)_{i, j=1}^{N}$ such that $f_{j}^{\prime}=\sum_{i=1}^{N} f_{i} \ell_{i j}$ and $\underline{u}^{\prime}(t)=K \underline{u}(t)$ where $\left(\left(\ell_{i j}\right)\right)_{i, j=1}^{N}=K^{-1}$.

Proof. Let $s$ be fixed $Y(t, s)=E\left(x(t) \mid \mathscr{F}_{s}\right)=\sum_{i=1}^{N} f_{i}(t) u_{i}(s)$ for $s<t$ in view of (0.2) (iii) and (iv). Now from (0.2) (i) and (ii) the first part follows. To prove the uniqueness we note $E\left(x(\tau) \mid \mathscr{F}_{s}\right)=\sum_{i=1}^{N} f_{i}(\tau) u_{i}(s)$ gives for any $s \leq t_{1}<t_{2}<\cdots<t_{N}, \underline{u}(s)=\Phi^{-1}(t) \underline{Y}(t, s)$ where $\Phi(t)=$ $\left(\left(f_{i}\left(t_{j}\right)\right)\right)_{i, j=1}^{N}$ and $\underline{Y}(t, s)=\left(Y\left(t_{1}, s\right), \cdots, Y\left(t_{n}, s\right)\right)^{*}$. Also $\underline{u}^{\prime}(s)=\Phi^{\prime-1}(t) \underline{Y}(t, s)$, where $\Phi^{\prime}(t)=\left(\left(f_{i}^{\prime}\left(t_{j}\right)\right)\right)_{i, j=1}^{N}$. Hence we have $\underline{u}(s)=\Phi^{-1}(\underline{t}) \Phi^{\prime}(t) \underline{u}^{\prime}(s)$. It therefore suffices to prove $\Phi^{-1}(t) \Phi^{\prime}(t)$ is independent of $t$. Let $\underset{\sim}{s}<t$ (that is, $s_{N}<t_{1}$ ). Choose $s^{\prime}<s_{1}$. Then we get

$$
\underline{u}\left(s^{\prime}\right)=\Phi^{-1}(s) \Phi^{\prime}(s) \underline{u}^{\prime}\left(s^{\prime}\right) .
$$

And

$$
\underline{u}\left(s^{\prime}\right)=\Phi^{-1}(t) \Phi^{\prime}(t) \underline{u}^{\prime}\left(s^{\prime}\right) .
$$

This implies $\Phi^{-1}(\underline{s}) \Phi^{\prime}(\underline{s})=\Phi^{-1}(\underset{t}{t}) \Phi^{\prime}(t)$ for any $\underline{s}<t$ so that $\Phi^{-1}(t) \Phi^{\prime}(t)$ is independent of $t$. Let $K=\Phi^{\prime-1}(t) \Phi(t)$. Then we get

$$
\begin{aligned}
x(t) & =\sum_{i=1}^{N} f_{i}^{\prime}(t) u_{i}^{\prime}(t) \\
& =\left(f_{1}(t), \cdots, f_{N}(t)\right) K^{-1} K \underline{u}(t) \\
& =\left(f_{1}(t), \cdots, f_{N}(t)\right) K^{-1} \underline{u}^{\prime}(t) .
\end{aligned}
$$

Also $x(t)=\left(f_{1}^{\prime}(t), \cdots, f_{N}^{\prime}(t)\right) \underline{u}^{\prime}(t)$, giving, by linear independence of $\left\{u_{1}^{\prime}(t)\right.$, $\left.\cdots, u_{N}^{\prime}(t)\right\}$, the result.

2. In this section we examine the Hida-Cramér multiplicity ([1], [5]) of GSP of the form (0.1) satisfying (0.2). In view of (0.2) (iv) we get that the multiplicity of such processes is equal to the multiplicity of a non-degenerate $N$-variate Martingale $\{\underline{u}(t), t \in \boldsymbol{R}\}$. Hence in view of ([10], p. 12) we get the following theorem immediately. 
2.1 THEOREM. The multiplicity of $N$-ple Markov process of the form (0.1) satisfying $(0.2)$ is at most $N$.

The question raised by Lévy was whether such $N$-ple Markov processes have multiplicity one. We give an example of an $N$-ple Markov process of multiplicity $N$. Naturally we use Theorem 1.10 and present the process in terms of its Lévy representation with the functions $\left\{f_{i}(t)\right\}_{i=1}^{N}$, $N-1$ times differentiable. Choose for each $i$ a continuously $N-1$ times differentiable function $f_{i}$. Assume that $f_{i}$ satisfies the following condition $(W), W_{k}\left(f_{1}, \cdots, f_{k}\right) \neq 0, k=1,2, \cdots, N$ where $W_{k}(k=1,2, \cdots, N)$ denote the Wronskian of order $k$. For the existence of such systems see Karlin ([7], p. 276). Also it follows from ([7], pp. 276-277) that $\left.\left.\operatorname{det}\left(\left(f_{i}\left(t_{j}\right)\right)_{i, j=1}^{N}\right)\right)\right) \neq 0$ for any $N$-points $t_{1}<\ldots<t_{N}$. Let $\left\{\xi_{i}^{(k)}, k= \pm 1\right.$, $\pm 2, \cdots, i=1, \cdots, N\}$ be a Gaussian system of independent random variables with mean zero and $\sum_{k=-\infty}^{+\infty} E\left(\xi_{i}^{(k)}\right)^{2}<\infty$ for $i=1,2, \cdots, N$, with $E\left(\xi_{i}^{(k)}\right)^{2}>0 . \quad x(t)=\sum_{i=1}^{N} f_{i}(t) \sum_{k \leq t} \xi_{i}^{(k)}$ where the infinite series converges a.e. under the assumptions. For $m-1 \leq t<m(m=0, \pm 1, \pm 2, \cdots)$ we get $x(t)=\sum_{i=1}^{N} f_{i}(t) u_{i}(m-1)$ where $u_{i}(t)=\sum_{k \leq t} \xi_{i}^{(k)}$. This implies $x$ is $N-1$ times differentiable in $q \cdot m^{4)}$ in $m-1 \leq t<m$. Now since $W_{N}\left(f_{1}, \cdots, f_{N}\right) \neq 0$ we get $u_{i}(m-1) \in H(x: t)$. But $u_{i}(t)=u_{i}(m-1)$ giving $u_{i}(t) \in H(x: t)(i=1,2, \cdots, N)$ for $m-1 \leq t<m(m=0, \pm 1, \cdots)$. This implies that $H(\underline{u}: t) \subseteq H(x: t), \forall t$. Clearly $H(x ; t) \subseteq H(\underline{u}: t)$. Properties (ii) and (iii) on the Lévy representation follow from definition $u_{i}(t)$ and mutual independence of $\left\{\xi_{i}^{(k)}\right\}$. The multiplicity of $\{x(t), t \in \boldsymbol{R}\}$ is therefore the same as $\{\underline{u}(t), t \in \boldsymbol{R}\}$. But multiplicity of $\underline{u}$ is equal to dimension of $H(\underline{u}: m) \theta H(\underline{u}: m-1)=N$.

2.2 Remark. (i) In the above example chosing for all $k, E\left(\xi_{i}^{(k)}\right)^{2}=0$ for $i=j+1, \cdots, N(1 \leq j \leq N)$ we get that multiplicity of $x$ is equal to $j,(1 \leq j \leq N)$.

(ii) Professor Hida informed me that Professor Hitsuda had an example of a 2-ple of multiplicity 2 , however $\left\{f_{1}(\cdot), f_{2}(\cdot)\right\}$ were chosen non-differentiable.

3. Motivated by the work of Lévy ([8], 4.7, p. 158) we call the representation of a process of the form

$$
x(t)=\sum_{i=1}^{N} f_{i}(t) u_{i}(t)
$$

4) At $\mathrm{m}-1$ the right derivatives are considered. 
where $\{\underline{u}(t), t \in \boldsymbol{R}\}$ is an $N$-variate Martingale, a Goursat representation (GR). We say that the GR is proper if $H(x: t)=H(\underline{u}: t)$. We now examine conditions on $x, f_{i}$ and $u_{i}$ in order that the GR is proper.

3.2 THEOREM. Let (i) the GSP $\{x(t), t \in \boldsymbol{R}\}$ have a GR, (ii) $x$ be $N-1$ times differentiable in quadratic mean and (iii) $\mathscr{F}_{t-0}(\underline{u})=\mathscr{F}_{t}(\underline{u})$. (iv) Each $f_{i}$ is $N-1$ times continuously differentiable and $W_{N}\left(f_{1}, \cdots, f_{N}\right)$ $\neq 0$. Then the GR is proper.

Proof. We have

$$
E\left(x(t) \mid \mathscr{F}_{s}(\underline{u})\right)=\sum_{i=1}^{N} f_{i}(t) u_{i}(s) \quad \text { for } s<t .
$$

Hence $E\left(x(t) \mid \mathscr{F}_{s}(\underline{u})\right)$ is $N-1$ times continuously differentiable in $t$. This implies that

(3.3) $\frac{\partial^{k}}{\partial t^{k}} E\left(x(t) \mid \mathscr{F}_{s}(\underline{u})\right)=\sum_{i=1}^{N} f_{i}^{(k)}(t) u_{i}(s) \quad$ for $k=0, \cdots, N-1$ and $s<t$.

By (3.3) and assumption (ii) we get that

$$
\frac{\partial^{k}}{\partial t^{k}} E\left(x(t) \mid \mathscr{F}_{s}\right)=E\left(x^{(k)}(t) \mid \mathscr{F}_{s}\right) \quad \text { for } k=0,1,2, \cdots, N-1
$$

where $x^{(k)}(t)$ denotes the $k^{t n} q \cdot m$ derivative of $x(t)$. Thus we get

$$
E\left(x^{(k)}(t) \mid \mathscr{F}_{s}(\underline{u})\right)=\sum_{i=1}^{N} f_{i}^{(k)}(t) u_{i}(s)
$$

Hence

$$
E\left(x^{(k)}(t) \mid \mathscr{F}_{s}(\underline{u})\right)=E\left(\sum_{i=1}^{N} f_{i}^{(k)}(t) u_{i}(t) \mid \mathscr{F}_{s}(\underline{u})\right) \quad \text { for } s<t
$$

This implies $E\left(x^{(k)}(t) \mid \mathscr{F}_{t-0}(\underline{u})\right)=E\left(\sum_{i=1}^{N} f_{i}^{(k)}(t) u_{i}(t) \mid \mathscr{F}_{t-0}(\underline{u})\right)$ giving by condition (iii)

$$
x^{(k)}(t)=\sum_{i=1}^{N} f_{i}^{(k)}(t) u_{i}(t), k=1,2, \cdots, N-1 .
$$

Now condition (iv) and (ii) conclude the proof.

3.7 Remark. Observe that if $u_{i}(t)=\int_{-\infty}^{t} g_{i}(u) B(d u)$ with $B$ representing the Brownian motion on the line and for each interval $(a, b]$ there exists at least one $g_{i} \neq 0$ on $(a, b]$, then $H(\underline{u}: t)=H(B: t)$. This 
can be seen as follows. Since $H(\underline{u}: t) \subseteq H(B: t)$. Suppose there is $y \in$ $H(B: t)$ orthogonal to $u_{i}(\tau)$ for $\tau \leq t$ and $i=1,2, \cdots, N$. Since $y=$ $\int_{-\infty}^{t} h(u) B(d u)$ and $y \perp H(\underline{u}: t)$ implies that $\int_{-\infty}^{\tau} h(u) g_{i}(u) d u=0$ for all $i=1,2, \cdots, N$ and $\tau \leq t$. But this implies for all $i, h(u) g_{i}(u)=0$ a.e. on $(-\infty, t]$, giving $h(u)=0$. Since for Brownian motion $H(B: t-0)=$ $H(B: t)$ we get $H(\underline{u}: t)=H(\underline{u}: t-0)$ in this case.

From the above remark, Theorem 3.2 has the following corollary.

3.8 CoRollary. Let $x(t)=\int_{-\infty}^{t} \sum_{i=1}^{N} f_{i}(t) g_{i}(u) B(d u)$ with $x, f_{i}^{\prime}$ 's as in Theorem 3.2 and $g_{i}$ 's as in Remark 3.7. Then (i) $x$ has a proper GR. (ii) $x$ has multiplicity one.

The proof of (i) is clear once we observe that $\underline{u}(t)=\left(u_{1}(t), \cdots, u_{N}(t)\right)^{*}$, where $u_{i}(t)=\int_{-\infty}^{t} g_{i}(u) B(d u)$ is $N$-variate Martingale since under the assumption of Remark 3.7, $H(\underline{u}: t)=H(B: t)=H(x: t)$ from which (ii) also follows.

We note that in view of [2], the GS Process occurring as solution of the $N^{t h}$ order stochastic differential equation with input Brownian motion $B$ given by $L_{t} x=B(d u) ; x(0)=0$ has the form

$$
x(t)=\int_{0}^{t} G(t, u) B(d u)
$$

where $G(t, u)$ is the Green's function of $L_{t}$. We therefore get

$$
x(t)=\int_{0}^{t} \sum_{i=1}^{N} f_{i}(t) g_{i}(u) B(d u)
$$

where $\left\{f_{i}(t)\right\}_{i=1}^{N}$ is a fundamental system of solutions of $L_{t} f=0$ and $\left\{g_{i}(\cdot)\right\}_{i=1}^{N}$ is a fundamental system of solutions of $L_{u}^{*} g=0$ where $L_{u}^{*}$ denotes the formal adjoint. Here we assume $W_{N}\left(f_{1}, \cdots, f_{N}\right) \neq 0$. In view of Corollary 3.8 and Remark 3.7. If $\left\{g_{i}\right\}$ satisfies the condition of Remark 3.7 one has $H(x: t)=H(u: t)=H(B: t)$. Hence the $x$ has proper GR. Also in this case $H(x: t-0)=H(x: t)$. Therefore $x$ satisfies condition of Theorem 1.1. We also know that since $W_{N}\left(f_{1}, \cdots, f_{N}\right) \neq 0,\left\{g_{1}, g_{2}, \cdots, g_{N}\right\}$ are linearly independent. Thus using Theorems 1.1, 1.2, Corollary 3.8, one deduces the following theorem

3.9 THEOREM. Let $x(t)$ be the solution of $N^{t h}$ order stochastic differential equations with input Brownian motion; viz, $x(0)=0$ and 
$L_{t} x=B(d u)$. Let $\left\{g_{i}\right\}$ satisfy condition of Remark 3.7. Then $x(t)$ is $N$-ple Markov iff $\operatorname{det}\left(\left(f_{i}\left(t_{j}\right)\right)_{i, j=1}^{N} \neq 0\right.$.

We say that a set $\left\{f_{1}, f_{2}, \cdots, f_{N}\right\}$ of $N-1$ times continuously differentiable functions satisfies property $(W)$ if $W_{k}\left(f_{1}, \cdots, f_{K}\right) \neq 0, k=$ $1,2, \cdots, N$.

The following lemma essentially due to Karlin ([7], p. 56) is now needed to obtain structure of $f_{i}$ satisfying conditions of the above theorem.

3.10 Lemмa. Let $\left\{f_{1}, f_{2}, \cdots, f_{N}\right\}$ be a set of $(N-1)$-times continuously differentiable real valued functions defined on $[0, \infty)$ such that $W\left(f_{1}, f_{2}, \cdots, f_{N}\right) \neq 0$ on $[0, T]$, for each $T$ finite. If for $0 \leq t_{1}<t_{2}<$ $\cdots<t_{N} \leq T$, $\operatorname{det}\left\{f_{i}\left(t_{j}\right)\right\}_{i, j=1,2, \cdots, N} \neq 0$. Then there exists a non-singular constant $N \times N$ matrix $B$ with $\left\{b_{i j}\right\}_{i, j=1,2 \ldots, N}$ such that the functions $h_{i}(t)$ $=\sum_{j=1}^{N} b_{i j} f_{i}(t)(i=1,2, \cdots, N)$ have the property $(W)$.

Proof of the lemma, except for trivial modification, is the same as in Karlin ([7], p. 57) and hence is omitted.

3.11 Remark. We note that if $\left\{f_{1}, \cdots, f_{N}\right\}$ is a fundamental system of solutions to the non-degenerate differential equation $L_{u} f=0$ and in addition $\operatorname{det}\left(\left(f_{i}\left(t_{j}\right)\right)\right) \neq 0$ for $0 \leq t_{1}<\cdots<t_{N} \leq T$ (for each $T$ finite), then in view of the above lemma we can choose another fundamental system $\left\{h_{1}, \cdots, h_{N}\right\}$ satisfying condition $(W)$. Thus we obtain from Theorem 3.9 and Lemma 3.10 the following striking theorem.

3.12 THEOREM. Let $x(t)$ be the solution of the stochastic differential equation $L_{t} x=B(d t)$ with $x(0)=0$ and suppose the fundamental system $\left\{g_{1}, \cdots, g_{N}\right\}$ of solutions of $L_{u}^{*} g=0$ satisfy condition of Remark 3.7. Then the following conditions are equivalent

(i) $x$ is N-ple Markov.

(ii) The operator $L_{u}$ is factorable in the sense of Ince ([6], p. 120).

Proof. In view of ([6], p. 120) we note that (ii) is equivalent to $L_{u} f=0$ having a fundamental system of solutions satisfying condition $(W)$. Equivalence (i) to this is obvious from Theorem 3.9 and Lemma 3.10 .

The functions $\left\{h_{1}, h_{2}, \cdots, h_{N}\right\}$ described in Lemma 3.10 can be explicitly written in terms of finite systems of non-vanishing functions (see Karlin [7], p. 276) in terms of iterated indefinite integrals. From this the following lemma is immediate. 
3.13 LEMma. The system $\left\{h_{1}, \cdots, h_{N}\right\}$ satisfies condition $(W)$ iff the fundamental system $\left\{K_{1}, \cdots, K_{N}\right\}$ of solutions to the formal adjoint equation $L_{u}^{*} f=0$ satisfy condition $(W)$.

Here $K_{j}(s)=W_{j}(s) / W\left(h_{1}, \cdots, h_{N}\right)(s)(j=1,2, \cdots, N)$, where $W_{j}(s)$ is the $N \times N$ determinant having the same elements as in $W_{N}\left(h_{1}, \cdots, h_{N}\right)(s)$ except with $(0, \cdots, 0,1)$ as its $j$ th column.

In view of the above lemma, Theorem 3.13 can be interpreted as strengthening of Lévy-Hida theorem (Theorem II.7, [5], [8], p. 159) in the sense of providing a partial converse to his theorem. This follows since if a system satisfies condition $(W)$ then $g_{i}$ satisfy of Remark 3.7 and since, if $\left\{f_{i}\right\}_{i=1}^{N}$ and $\left\{g_{i}\right\}_{i=1}^{N}$ are given by (II.29) and (II.25) of [5] then

$$
x(t)=\sum_{i=1}^{N} \int_{-\infty}^{t} f_{i}(t) g_{i}(u) B(d u)
$$

satisfies $L_{t} x=B(d u)$ with $L_{t}$ as in ([5], p. 138). We also remark that Theorem 3.12 provides a rigorous proof of Lévy's claim ([8], $2^{\circ}$, p. 159).

Note added in Proof. After this work was with the referee, Prof. L. Pitt informed me of some of his work which has several points of contact with this one.

\section{REFERENCES}

[1] H. Cramér, Stochastic processes as curves in Hilbert space, Teoria veryatnosti i ee premenenie IX, 2, (1964), 193-204.

[2] C. L. Dolph and M. A. Woodbury, On the relation between Green's functions and covariances of certain stochastic processes and its application to unbiased linear predictor, Trans. Amer. Math. Soc. 72 (1952), 519-550.

[ 3 ] J. L. Doob, Stochastic Processes, John Wiley, New York, 1953.

[4] H. Dym and H. P. McKean, Jr., Applications of De Branges spaces of integral functions to the prediction of stationary Gaussian processes, Ill. J. Math. 14 (1970), 299-343.

[5] T. Hida, Canonical representations of Gaussian processes and their applications, Mem. Coll. Sci. Kyoto A33 (1960), 109-155.

[ 6 ] E. L. Ince, Ordinary Differential Equations, Dover, New York, 1926.

[ 7 ] S. Karlin, Total positivity, Stanford University Press, Stanford, 1968.

[ 8 ] P. Lévy, A special problem of Brownian motion, and a general theory of Gaussian random functions, Proc. Third. Berk. Symp. Math. Stat. and Prob. II (1956), $133-175$.

[ 9 ] P. Lévy, Fonctions linearement Markoviennes d'ordre n, Math. Japonicae 4 (1957), 113-121.

[10] V. Mandrekar, On multivariate wide-sense Markov processes, Nagoya Math. J. 33 (1968), 7-19. 
[11] V. Mandrekar, On multiple Markov process of T. Hida and their prediction, Preprint 1971.

Michigan State University

E. Lansing

Mich. 48823 